\title{
Serologic Screening of Celiac Disease in Patients With Type 1 Diabetes
}

\author{
Eman M. Albatayneh ${ }^{\mathrm{a}, \mathrm{f}}$, Nedal A. Alnawaiseh ${ }^{\mathrm{b}}$, Sameeh A. Al-Sarayreh ${ }^{\mathrm{c}}$, Yousef M. Al-saraireh ${ }^{\mathrm{d}}$, \\ Enas M. Al-Zayadnehe, Mohammad A. Abu-lobbad ${ }^{\mathrm{a}}$
}

\begin{abstract}
Background: Type 1 diabetes mellitus (T1DM) is an autoimmune disorder associated with increased risk of additional autoimmune diseases (ADs) as celiac disease (CD). The aim of this study was to investigate the prevalence of CDs in a population of diabetic children along with the presence of any suggestive clinical signs and symptoms of $\mathrm{CD}$ and any effects of $\mathrm{CD}$ on the patients' diabetic control and growth.
\end{abstract}

Methods: Data from diabetic patients in South Jordan attending the diabetic clinic in the military hospital between 2014 and 2015 were collected. The frequency of CD was calculated. A $t$-test comparison between CD-seropositive and seronegative diabetic patients, the unadjusted and adjusted odds ratios (ORs) for age, gender, baseline glycated hemoglobin $(\mathrm{HbAlc})$, cholesterol and glucose levels, associated diseases, and body mass index with their $95 \%$ confidence intervals (CIs) were evaluated.

Results: One hundred thirty-eight patients with T1DM (median age 16.5 years; 74 females) were recruited. The results showed that $\mathrm{CD}$ was in $(6.5 \%)$ of the patients. The body weight, body mass index, baseline $\mathrm{HbA} 1 \mathrm{c}$ and cholesterol levels were significantly different between seropositive and seronegative patients $(\mathrm{P}<0.05)$. Adjusted ORs for the presence eczema and family history of T1DM in seropositive CD were 32.66 (95\% CI: $2.68-398.85, \mathrm{P}=0.0006)$, and 5.79 (95\% CI: $1.07-31.33, \mathrm{P}=0.041$ ), respectively. While the adjusted odd ratio of having high $\mathrm{HbA} 1 \mathrm{c}$ and high cholesterol levels in seropositive were $1.76(95 \% \mathrm{CI}: 1.13-2.74, \mathrm{P}=0.01)$ and $1.02(95 \% \mathrm{CI}$ : $1.006-1.031, \mathrm{P}<0.001)$ respectively.

Manuscript submitted April 5, 2018, accepted April 23, 2018

${ }^{a}$ Department of Microbiology and Immunology, Faculty of Medicine, Mutah University, Al-Karak, Jordan

${ }^{b}$ Department of Public Health, Faculty of Medicine, Mutah University, AlKarak, Jordan

'Department of Biochemistry and Molecular Biology, Faculty of Medicine, Mutah University, Al-Karak, Jordan

${ }^{\mathrm{d} D e p a r t m e n t}$ of Pharmacology, Alkaptonuria Research Office, Faculty of Medicine, Mutah University, Al-Karak, Jordan

eDepartment of Pediatrics, Faculty of Medicine, Mutah University, Al-Karak, Jordan

${ }^{\mathrm{f} C}$ Corresponding Author: Eman Albatayneh, Department of Microbiology and Immunology, Faculty of Medicine, Mutah University, Al-Karak, Jordan. Email: emanbatayneh@yahoo.com,

doi: https://doi.org/10.14740/jem507w
Conclusions: These findings suggest a need for a careful surveillance of $\mathrm{CD}$ in patients with T1DM. This may help in improving the diabetic control and growth control, and avoidance of complications.

Keywords: Type 1 diabetes mellitus; Celiac disease; Autoimmune disease; Diabetic control

\section{Introduction}

Type 1 diabetes mellitus (T1DM) is a common autoimmune disease in children. The risk for other autoimmune disorders is increased in children suffering from T1DM. Recent evidence indicates that celiac disease (CD) is an autoimmune disease characterized by immune-mediated damage to the mucosa of the small intestine [1] and is triggered by the ingestion of gluten in genetically susceptible individuals. Although CD was once believed to be rare, recent studies have shown an increasing incidence of $\mathrm{CD}$ in both pediatric and adult populations [2]. However, diarrhea and weight loss which were believed to be the hallmarks of $\mathrm{CD}$, are observed less commonly in recently diagnosed cases.

The association between T1DM and CD has been demonstrated in children. There is also evidence of common genetic basis for disease expression, as both diseases are associated with the major histocompatibility complex class II antigen DQ2 encoded by the alleles DQA1*501 and DQB1*201 and seven shared non-human leucocyte antigen (HLA) loci [3]. Globally, the estimated prevalence of CD in the general population is around $1 \%$ [4], but it increases by 5 - to 7 -fold when concomitant with T1DM [5]. This variation in coexistence is determined by geographical/genetic predisposition and diabetes duration. The prevalence of CD in association with T1DM, as estimated in two studies conducted in north India $[6,7]$ ranges from $11 \%$ to $17 \%$. However, according to the European studies, the prevalence of $\mathrm{CD}$ in diabetic populations ranges from $1.5 \%$ to $4.6 \%$ in children, and $2 \%$ to $4.1 \%$ in adults $[8,9]$.

$\mathrm{CD}$ has protean manifestations, but majority of patients with coexisting T1DM do not have classical gastrointestinal symptoms of CD. They are either asymptomatic or manifest with atypical features such as short stature, refractory anemia, delayed puberty, osteopenia and other autoimmune disorders such as thyroiditis and autoimmune hepatitis [5]. Clinical findings suggestive of CD in T1DM include unpredictable blood 
Table 1. Demographic Data $($ Total $=138$, Male $=64$, Female $=74)$

\begin{tabular}{lllll}
\hline Variable & Minimum & Maximum & Mean & Standard deviation \\
\hline Age (years) & 1.00 & 26.00 & 16.53 & 5.62 \\
Weight $(\mathrm{kg})$ & 7.00 & 133.00 & 62.52 & 23.38 \\
Length $(\mathrm{cm})$ & 75.00 & 186.00 & 1.57 & 20.30 \\
BMI & 7.77 & 48.29 & 24.48 & 7.05 \\
HbAlc value (\%) & 6.40 & 12.80 & 8.67 & 1.56 \\
Triglycerides value (mg/dL) & 48 & 430.00 & 2.10 & 136.71 \\
Cholesterol value (mg/dL) & 68.00 & 400.00 & 2.15 & 88.17 \\
Fasting glucose value (mg/dL) & 51.00 & 450.00 & 1.20 & 52.33 \\
Hemoglobin level $(\mathrm{Hb})$ & 7.67 & 17.00 & 12.14 & 1.68 \\
\hline
\end{tabular}

glucose levels, recurrent episodes of hypoglycemia and growth failure [10]. These symptoms are often attributed to poor glycemic control. Failure to recognize coexisting CD may predispose the affected individuals to increased risk of growth failure, osteoporosis, infertility and gastrointestinal lymphoma [11]. It is also speculated that continuous exposure to gluten may facilitate development and progression of other autoimmune diseases apart from CD [12]. Hence, it is important to actively screen for CD in patients with T1DM at the time of diagnosis, as well as during subsequent follow-ups. This will help optimize insulin therapy, achieve good glycemic control and reduce the risk of complications due to both T1DM and CD [13].

Even though the gold standard for the diagnosis of CD is duodenal biopsy, serologic screening for $\mathrm{CD}$ has been proven beneficial. According to the Agency for Healthcare Research and Quality (AHRQ) report, using histology as reference standard, IgA-anti-tissue transglutaminase (tTG) tests appear highly accurate to diagnose $\mathrm{CD}$, yet it is not invasive $[14,15]$.

The prevalence of celiac disease among children in Jordan was examined in 2010 and was reported at 1\% [16]. However, a screening study on CD in T1DM patients has never been conducted.

Thus, the aim of the present study was to establish the prevalence of celiac disease among patients with T1DM in South Jordan, along with the presence of any suggestive clinical signs and symptoms of $\mathrm{CD}$ and any effects of $\mathrm{CD}$ on the patients' diabetic control and growth.

\section{Methods}

After obtaining the approval from the scientific and ethics committees, 138 patients with T1DM attending the diabetic clinic in the military hospital were interviewed. Once written consent was obtained from the patients or their parents, the patients completed a questionnaire focusing on their age, weight, height, diabetes control, and symptoms and signs of celiac disease, such as heartburn, weakness, eczema, joint pain, numbness, depression, menstrual cycle abnormality, lactose intolerance and growth retardation, personal history and family history of $\mathrm{CD}$, osteoporosis, chronic anemia, depres- sion, chronic eczema, thyroid dysfunction, infertility, vitiligo, irritable bowel disease, etc. In addition, serum samples were obtained from each participant and were analyzed in duplicates for IgA antibodies to human tTG using enzyme linked immunosorbent assay (ELISA) (Orgentec Diagnostika $\mathrm{GmbH}$, Mainz, Germany). The test has a lower detection limit of 1.0 $\mathrm{U} / \mathrm{mL}$ and $10 \mathrm{U} / \mathrm{mL}$ was the cut-off point for a positive result. Patients positive for the antibody were asked to repeat the test. Among the seropositive group, those with anti-tTG levels $\geq 3$ times the reference cut-off were advised to undergo endoscopic biopsy, as per the European Society for Pediatric Gastroenterology, Hepatology, and Nutrition criteria [17]. Serum IgA levels were measured in children with negative celiac serology to detect IgA deficiency. Other blood tests, such as $\mathrm{CBC}$, hemoglobin ( $\mathrm{Hb}), \mathrm{FBS}, \mathrm{HbA} 1 \mathrm{c}$ and biochemistry, were obtained from the patients' records.

\section{Statistical analyses}

The data were analyzed using SPSS software version 16.0 (SPSS Inc., Chicago, USA). Continuous variables were summarized as mean $\pm \mathrm{SD}$, while categorical variables were reported as frequencies and percentages. Proportion of seropositive children with confirmed $\mathrm{CD}$ was calculated and 95 percent confidence interval (CI) was determined [18]. Simple logistic regression [19] was applied to study the association of variables (like age, sex, height, body mass index (BMI), FBS, cholesterol, $\mathrm{Hb}$, triglycerides, HbA1c levels and CD-related symptoms) in T1DM with celiac seropositivity. The odds ratios (ORs) with P value were calculated.

\section{Results}

\section{Demographic data}

Table 1 shows the demographic characteristics of the study sample, comprising of 64 diabetic males and 74 diabetic females. The sample mean age was 16.5 years and the average T1DM duration was 48 months. Their fasting blood sugar was relatively high. 


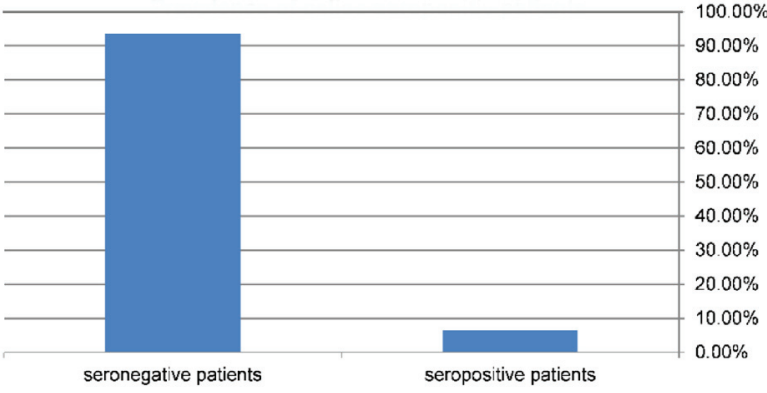

Figure 1. Prevalence of diabetic patients that have celiac seropositive results $=6.5 \%(9 / 138)$.

\section{Prevalence of celiac disease}

Records of 138 children and adolescents (64 males and 74 females) with T1DM were reviewed. The mean age at enrolment was $16.9 \pm 5.3$ and $16.2 \pm 5.9$ years for boys and girls, respectively. Nine children $(6.5 \%$; $95 \%$ CI: 3.47 - 11.93) were seropositive for CD with IgA anti-tTG levels $>3$ times the reference cut-off, with a higher rate in females $(6.760 \%$; $95 \%$ CI: 2.92 - 14.86) compared to males $(6.25 \%$; 95\% CI: 2.46 15.00) (Fig. 1,2). However, the difference was not statistically significant $(\mathrm{P}>0.05)$. None of the participants with negative serology were found to be IgA deficient.

\section{Characteristics of seropositive patients}

The mean age at presentation was significantly lower among seropositive compared to seronegative individuals $(\mathrm{P}=0.024)$, and the mean weight and BMI values were lower among seropositive as compared to seronegative children $(\mathrm{P}$ value $=0.015$, 0.022, respectively). Higher baseline glycated hemoglobin (HbA1c) and cholesterol levels in seropositive patients were noted as well $(\mathrm{P}$ value $=0.007,0.001$, respectively $)($ Table 2$)$

\section{Diseases associate with celiac-seropositive patients}

The probability of having personal complaint of eczema or being diagnosed with eczema was significantly higher in CDpositive diabetic patients ( $\mathrm{P}=0.025$ and 0.006 , respectively).

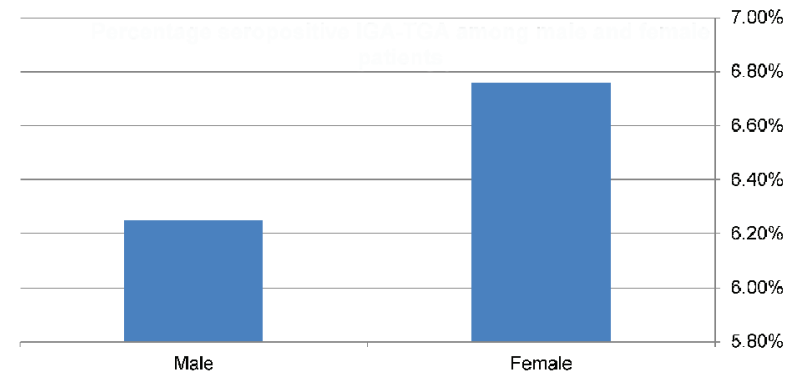

Figure 2. Prevalence of celiac-seropositive patients among males $(4 / 64)$ and females (5/74).
Table 2. The Significant Different Numerical Variables Between IgA Seropositive and Seronegative Diabetic Patients

\begin{tabular}{lllll}
\hline & IgA-TGA & Mean & SD & P value \\
\hline Age (years) & No & 16.81 & 5.56 & 0.024 \\
& Yes & 12.44 & 5.25 & \\
Weight $(\mathrm{kg})$ & No & 63.80 & 22.79 & 0.015 \\
& Yes & 44.22 & 25.29 & \\
HbA1c value (\%) & No & 8.57 & 1.51 & 0.007 \\
& Yes & 10.00 & 1.62 & \\
Cholesterol value (mg/dL) & No & 208.43 & 87.03 & 0.001 \\
& Yes & 308.89 & 36.56 & \\
BMI & No & 24.84 & 7.014 & 0.022 \\
& Yes & 19.30 & 5.56 & \\
\hline
\end{tabular}

Furthermore, the prevalence of family history of celiac disease and T1DM was greater in celiac-positive diabetic patients $(\mathrm{P}$ value $=0.004$, and 0.019, respectively) (Table 3). Stepwise binary logistic regression with backward entry with the IgATGA seropositive as the dependent variable, and all the previous significant characteristics shown in Table 3 as independent variables was used to determine a subset of characteristics that best predicts the presence of celiac disease within the target population (Table 4). The findings indicate that, among the children that are seropositive for celiac disease, personal complaints of eczema and family history of T1DM are about 32 and 5 times more frequent, respectively, compared to their healthy counterparts.

To identify numerical variables that are the best predictors of celiac disease, multiple logistic regression was used, in which the significant characteristics shown in Table 2 were treated as independent variables (Table 5). The findings indicate that, among the children that having celiac disease with diabetes, the rates for reported high $\mathrm{Hb} 1 \mathrm{AC}$ and cholesterol levels are about 1.76 and 1.02, respectively, as high as in their diabetic counterparts.

\section{Clinical features of $\mathrm{CD}$}

Typical clinical features of CD were analyzed in IgA TGApositive patients (Fig. 3). The common symptoms, such as abdominal fullness, diarrhea and weight loss, were present only in two patients, whereas the uncommon ones, including joint

Table 3. The Significant Difference of Categorical Variables Between IgA Seropositive and Seronegative Diabetic Patients

\begin{tabular}{ll}
\hline Variables & P value* \\
\hline Patient complain of eczema & 0.025 \\
Patient diagnosed as eczema & 0.006 \\
Family history of celiac disease & 0.004 \\
Family history of DM 1 & 0.019 \\
\hline
\end{tabular}

*Fisher exact $P$ value. 
Table 4. Logistic Regression Showing the Predictor Variable for Seropositive Diabetic Patients

\begin{tabular}{llllc}
\hline Categorical variable & P-value & AOR* & \multicolumn{2}{c}{ 95\% CI (AOR) } \\
\cline { 4 - 5 } & & & Lower & Upper \\
\hline Patient's diagnosis of eczema & 0.006 & 32.662 & 2.675 & 398.846 \\
Family history of T1DM & 0.041 & 5.794 & 1.072 & 31.328 \\
\hline
\end{tabular}

*Adjusted odd ratio.

Table 5. Linear Regression for Numerical Variables Associated With IgA Seropositivity

\begin{tabular}{lllll}
\hline Variable & AOR* & $\mathbf{9 5 \%}$ CI (AOR) & Chi-square & P value \\
\hline HbA1c & 1.76 & $1.134-2.741$ & 6.66 & 0.010 \\
Cholesterol & 1.018 & $1.006-1.031$ & 12.78 & $<0.001$ \\
\hline
\end{tabular}

*Adjusted odds ratios.

pain, were noted in six patients. General weakness and numbness was reported by five patients.

\section{Discussion}

In this study, the overall prevalence of $\mathrm{CD}$ in patients of all ages with T1DM was $6.5 \%$, which is about 6 times higher compared to that in the general population and was comparable to that reported in other studies conducted in Arab countries. The reported prevalence of CD in the T1DM patients from Saudi Arabia is 10\% [20], while 16.4\% was reported for Algeria $16.4 \%$ [21] and 11\% for Iraq [22]. The difference in the prevalence of CD across geographical locations was attributed to the variations in the distribution of the HLA genotypes. Furthermore, study designs were incompatible, as variability in population studied; population size, testing procedures, and the definition of a seropositive result were noted. In addition, the serological tests used to estimate the disease prevalence varied.

The sequence of appearance of T1DM and CD cannot be predicted. Pediatric patients with T1DM are not screened for $\mathrm{CD}$, even though overt diabetes and celiac disease can be ultimately diagnosed at approximately the same age [23]. The children who developed both diabetes- and CD-associated antibodies generated the two types of antibodies usually in a random order within a short time interval. One possibility is that the children with CD-associated antibodies seroconverted to positivity for diabetes-associated autoantibodies earlier than those who did not have CD-associated antibodies, so that celiac disease developed earlier than diabetes [24, 25]. Whereas, results differ clearly in another study, in which diabetes-associated autoantibodies developed earlier than celiac disease-associated antibodies [26] and the diagnosis of T1DM

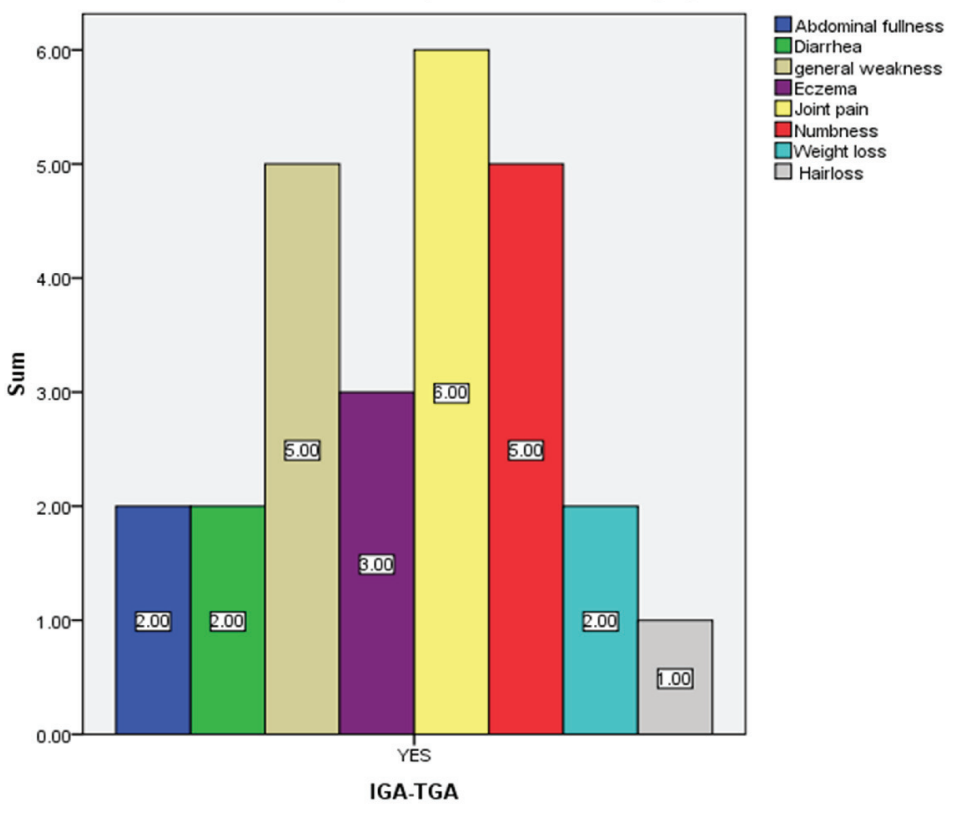

Figure 3. Number of celiac seropositive patients who have celiac-related symptoms. 
usually precedes CD [23]. In our study, the patients suffering from both $\mathrm{CD}$ and diabetes were younger than the patients affected by diabetes only, which may be explained by the fact of that CD-associated antibodies seroconverted to positivity for diabetes-associated autoantibodies earlier than having just diabetes autoantibodies [24].

The predominance of female gender among $\mathrm{CD}$ group in the present study has been observed previously [24, 25]. Authors of a multicenter study conducted in Italy demonstrated a positive association between female gender and the development of CD [27].

Studies on impact of $\mathrm{CD}$ on diabetes control and growth in children with T1DM have yielded conflicting results [21]. Our observation of poor diabetic control, a lower weight and a lower BMI in patients with coexisting T1DM and CD was in agreement with reports from Indian [6] and other studies [28]. In addition, our results showed that the coexisting hypercholesterolemia increased the odds of CD in children with T1DM, which was noted in many studies. Moreover, it may increase the risk of cardiovascular disease in children with T1DM and untreated CD [29]. In this study, the patients suffering from celiac disease showed a higher risk of having concomitant eczema. A similar association has been reported in extant literature $[30,31]$. One theory is that serum $\operatorname{IgG}$ antibodies reactive with different dietary proteins have been detected in a significant proportion of adult patients with $\mathrm{CD}$ and dermatitis herpetiformis. Moreover, atopic eczema and the protective antibody IgG4 anti-gliadin antibodies were less prevalent in the serum of patients with $\mathrm{CD}$ than in healthy controls, suggesting defective downstream switching of Ig heavy-chain genes in these conditions [32].

Screening high-risk groups may be beneficial. In our study, having family history of T1DM or CD was a good predictor for celiac disease. This finding is in line with results reported by other authors. For example one study showed that an increased prevalence of $\mathrm{CD}$ autoimmunity in the children of parents diagnosed with T1DM, and another study suggested a prevalence of CD of $3-7 \%$ of T1DM and $4 \%-10 \%$ of firstdegree family members $[33,34]$.

From a clinical perspective, our study showed that symptoms presented by our patients with $\mathrm{CD}$ cannot be used to predict the disease as some non-CD patients report these symptoms, so they were not specific to $\mathrm{CD}$ making diagnosis on purely clinical grounds more difficult.

Because the prevalence of $\mathrm{CD}$ is higher in patients with T1DM, screening programs for $\mathrm{CD}$ as part of routine care should be instituted. From a medical perspective, numerous advantages may be obtained from screening asymptomatic diabetic patients, including the potential for improved diabetic control, growth control and avoidance of extra-intestinal manifestations of $\mathrm{CD}$, notably osteopenia and malignancy [35, 36]. Moreover, by early screening, the effects of an additional chronic disease, such as $\mathrm{CD}$, on the quality of life in diabetic patients may be reduced. Unfortunately, we are not aware of any extant studies that address the psychosocial effects of CD in asymptomatic diabetic patients.

This study had several strengths, including that the serological results were confirmed by conducting the tests in triplicates. The shortcomings of this study may include its retro- spective design and variable duration of follow-up, as well as a small sample size, which would limit generalizability of our findings to other populations. Furthermore, we used serologybased prevalence study as the main estimate of CD in the studied population. This may result in false positive as well as false negative $\mathrm{CD}$ cases because the correlation between serological markers and small bowel biopsy is not $100 \%$. Even though we were able to identify silent CD-positive cases in an at-risk population by using rigorous and uniform diagnostic criteria for both T1DM and CD, clinical benefits of identifying all cases of $\mathrm{CD}$ remain controversial [37].

\section{References}

1. Farrell RJ, Kelly CP. Celiac sprue. N Engl J Med. 2002;346(3):180-188.

2. Catassi C, Gatti S, Fasano A. The new epidemiology of celiac disease. J Pediatr Gastroenterol Nutr. 2014;59(Suppl 1):S7-9.

3. Smyth DJ, Plagnol V, Walker NM, Cooper JD, Downes K, Yang JH, Howson JM, et al. Shared and distinct genetic variants in type 1 diabetes and celiac disease. N Engl J Med. 2008;359(26):2767-2777.

4. Fasano A, Berti I, Gerarduzzi T, Not T, Colletti RB, Drago S, Elitsur Y, et al. Prevalence of celiac disease in at-risk and not-at-risk groups in the United States: a large multicenter study. Arch Intern Med. 2003;163(3):286-292.

5. Gillett PM, Gillett HR, Israel DM, Metzger DL, Stewart L, Chanoine JP, Freeman HJ. High prevalence of celiac disease in patients with type 1 diabetes detected by antibodies to endomysium and tissue transglutaminase. Can J Gastroenterol. 2001;15(5):297-301.

6. Bhadada SK, Kochhar R, Bhansali A, Dutta U, Kumar PR, Poornachandra KS, Vaiphei K, et al. Prevalence and clinical profile of celiac disease in type 1 diabetes mellitus in north India. J Gastroenterol Hepatol. 2011;26(2):378381.

7. Agrawal RP, Rathore A, Joshi A, Changal H, Kochar DK. Prevalence of celiac disease in type 1 diabetes mellitus in North West Rajasthan, India. Diabetes Res Clin Pract. 2008;79(1):e15-16.

8. Savilahti E, Simell O, Koskimies S, Rilva A, Akerblom HK. Celiac disease in insulin-dependent diabetes mellitus. J Pediatr. 1986;108(5 Pt 1):690-693.

9. Koletzko S, Burgin-Wolff A, Koletzko B, Knapp M, Burger W, Gruneklee D, Herz G, et al. Prevalence of coeliac disease in diabetic children and adolescents. A multicentre study. Eur J Pediatr. 1988;148(2):113-117.

10. Mohn A, Cerruto M, Iafusco D, Prisco F, Tumini S, Stoppoloni O, Chiarelli F. Celiac disease in children and adolescents with type I diabetes: importance of hypoglycemia. J Pediatr Gastroenterol Nutr. 2001;32(1):37-40.

11. Goddard CJ, Gillett HR. Complications of coeliac disease: are all patients at risk? Postgrad Med J. 2006;82(973):705712 .

12. Toscano V, Conti FG, Anastasi E, Mariani P, Tiberti C, Poggi M, Montuori M, et al. Importance of gluten in the induction of endocrine autoantibodies and organ dysfunc- 
tion in adolescent celiac patients. Am J Gastroenterol. 2000;95(7):1742-1748.

13. Kordonouri O, Klingensmith G, Knip M, Holl RW, Aanstoot HJ, Menon PS, Craig ME, et al. ISPAD Clinical Practice Consensus Guidelines 2014. Other complications and diabetes-associated conditions in children and adolescents. Pediatr Diabetes. 2014;15(Suppl 20):270-278.

14. Hopper AD, Hadjivassiliou M, Hurlstone DP, Lobo AJ, McAlindon ME, Egner W, Wild G, et al. What is the role of serologic testing in celiac disease? A prospective, biopsy-confirmed study with economic analysis. Clin Gastroenterol Hepatol. 2008;6(3):314-320.

15. Shomaf M, Rashid M, Faydi D, Halawa A. Is the Diagnosis of Celiac Disease Possible Without Intestinal Biopsy? Balkan Med J. 2017;34(4):313-317.

16. Nusier MK, Brodtkorb HK, Rein SE, Odeh A, Radaideh AM, Klungland H. Serological screening for celiac disease in schoolchildren in Jordan. Is height and weight affected when seropositive? Ital J Pediatr. 2010;36:16.

17. Husby S, Koletzko S, Korponay-Szabo IR, Mearin ML, Phillips A, Shamir R, Troncone R, et al. European Society for Pediatric Gastroenterology, Hepatology, and Nutrition guidelines for the diagnosis of coeliac disease. J Pediatr Gastroenterol Nutr. 2012;54(1):136-160.

18. Clopper CJ, Pearson ES. The use of confidence or fiducial limits illustrated in the case of the binomial. Biometrika. 1934:26(4)404-413.

19. Hosmer DW Jr, Lemeshow S, Sturdivant RX. Applied logistic regression. John Wiley \& Sons. 2013.

20. Al-Hakami AM. Pattern of thyroid, celiac, and anti-cyclic citrullinated peptide autoantibodies coexistence with type 1 diabetes mellitus in patients from Southwestern Saudi Arabia. Saudi Med J. 2016;37(4):386-391.

21. Sud S, Marcon M, Assor E, Palmert MR, Daneman D, Mahmud FH. Celiac disease and pediatric type 1 diabetes: diagnostic and treatment dilemmas. Int J Pediatr Endocrinol. 2010;2010:161285.

22. Mansour AA, Najeeb AA. Coeliac disease in Iraqi type 1 diabetic patients. Arab J Gastroenterol. 2011;12(2):103105.

23. Larsson K, Carlsson A, Cederwall E, Jonsson B, Neiderud J, Jonsson B, Lernmark A, et al. Annual screening detects celiac disease in children with type 1 diabetes. Pediatr Diabetes. 2008;9(4 Pt 2):354-359.

24. Simell S, Hoppu S, Simell T, Stahlberg MR, Viander M, Routi T, Simell V, et al. Age at development of type 1 diabetes- and celiac disease-associated antibodies and clinical disease in genetically susceptible children observed from birth. Diabetes Care. 2010;33(4):774-779.

25. Valerio G, Maiuri L, Troncone R, Buono P, Lombardi F, Palmieri R, Franzese A. Severe clinical onset of diabetes and increased prevalence of other autoimmune diseases in children with coeliac disease diagnosed before diabetes mellitus. Diabetologia. 2002;45(12):1719-1722.

26. Hummel S, Hummel M, Banholzer J, Hanak D, Mollenhauer U, Bonifacio E, Ziegler AG. Development of autoimmunity to transglutaminase $\mathrm{C}$ in children of patients with type 1 diabetes: relationship to islet autoantibodies and infant feeding. Diabetologia. 2007;50(2):390-394.

27. Cerutti F, Bruno G, Chiarelli F, Lorini R, Meschi F, Sacchetti C, Diabetes Study Group of the Italian Society of Pediatric E, et al. Younger age at onset and sex predict celiac disease in children and adolescents with type 1 diabetes: an Italian multicenter study. Diabetes Care. 2004;27(6):1294-1298.

28. Amin R, Murphy N, Edge J, Ahmed ML, Acerini CL, Dunger DB. A longitudinal study of the effects of a gluten-free diet on glycemic control and weight gain in subjects with type 1 diabetes and celiac disease. Diabetes Care. 2002;25(7):1117-1122.

29. Salardi S, Maltoni G, Zucchini S, Iafusco D, Zanfardino A, Confetto S, Toni S, et al. Whole lipid profile and not only HDL cholesterol is impaired in children with coexisting type 1 diabetes and untreated celiac disease. Acta Diabetol. 2017;54(10):889-894.

30. Friedman M, Hare PJ. Gluten-Sensitive Enteropathy and Eczema. Lancet. 1965;1(7384):521-524.

31. Ohlsen BA. Acupuncture and a gluten-free diet relieve urticaria and eczema in a case of undiagnosed dermatitis herpetiformis and atypical or extraintestinal celiac disease: a case report. J Chiropr Med. 2011;10(4):294-300.

32. Barnes RM, Harvey MM, Blears J, Finn R, Johnson PM. IgG subclass of human serum antibodies reactive with dietary proteins. Int Arch Allergy Appl Immunol. 1986;81(2):141-147.

33. Murray JA. Celiac disease in patients with an affected member, type 1 diabetes, iron-deficiency, or osteoporosis? Gastroenterology. 2005;128(4 Suppl 1):S52-56.

34. Boudraa G, Hachelaf W, Benbouabdellah M, Belkadi M, Benmansour FZ, Touhami M. Prevalence of coeliac disease in diabetic children and their first- degree relatives in west Algeria: screening with serological markers. Acta Paediatr Suppl. 1996;412:58-60.

35. Freemark M, Levitsky LL. Screening for celiac disease in children with type 1 diabetes: two views of the controversy. Diabetes Care. 2003;26(6):1932-1939.

36. Holmes GK. Non-malignant complications of coeliac disease. Acta Paediatr Suppl. 1996;412:68-75.

37. Hoffenberg EJ, Emery LM, Barriga KJ, Bao F, Taylor J, Eisenbarth GS, Haas JE, et al. Clinical features of children with screening-identified evidence of celiac disease. Pediatrics. 2004;113(5):1254-1259. 